\title{
OPTICAL IMAGE-BASED DYNAMIC MANIPULATION OF AQUEOUS DROPLETS IMMERSED IN OIL MEDIUM
}

\author{
Sung-Yong Park ${ }^{1}$, Ting-Hsiang $W^{2}$, Kenneth $W^{1} i^{1}$, Sheraz Kalim ${ }^{3}$, Caitlin Callahan ${ }^{3}$, \\ Michael Teitell ${ }^{3}$ and Eric P.Y. Chiou ${ }^{1}$ \\ ${ }^{1}$ The Department of Mechanical and Aerospace Engineering, University of California at Los Angeles, \\ 43-147 Eng. IV, 420 Westwood Plaza, Los Angeles, CA 90095-1597, USA \\ ${ }^{2}$ The Department of Electrical Engineering, University of California at Los Angeles, USA \\ ${ }^{3}$ The Department of Pathology and Pediatrics, University of California at Los Angeles, USA
}

\begin{abstract}
We report on an optically reconfigurable microfluidic platform enabling large scale, parallel processing of oil-immersed aqueous droplets on a featureless photoconductive glass substrate using direct optical images. Aqueous droplets containing chemical and biochemical contents are manipulated by optical image-patterned virtual electrodes through dielectrophoretic (DEP) forces to perform various droplet manipulation functions including (1) continuous 2D transport, (2) droplet merging, (3) and parallel processing of sixteen droplets. Our platform promises a low cost, silicon-coated microfluidic system for large scale, multiplexed droplet-based biochemical analysis.
\end{abstract}

\section{INTRODUCTION}

Droplet-based microfluidic systems have the potential for high-throughput and speed chemical and biological analysis ${ }^{1-4}$. Thousands of highly uniform, isolated aqueous droplets containing chemical reagents can be generated in immiscible oil within seconds by flow-focusing devices. Applications such as fluorescent detection of millisecond chemical kinetics, laser Raman spectroscopic probing, and PCR reactions for high throughput DNA amplification have been demonstrated using droplet-based microfluidic devices ${ }^{5-7}$. A major difficulty associated with a droplet-based microfluidic system is in achieving active control of individual droplets confined in microchannels being driven by a continuous oil flow ${ }^{2-6}$. It has been shown that multiple droplets can be individually addressed in an open oil chamber using physically patterned electrodes, although complex wiring and interconnection issues arise for addressing numerically large droplet arrays ${ }^{8,9}$. To solve this problem, a microfluidic device integrating a high voltage CMOS driving circuit recently achieved active and parallel droplet control. However, this approach increases the fabrication cost of microfluidic devices, which are often preferred to be disposable ${ }^{10}$.

Alternatively, a light-induced manipulation mechanism has been widely investigated to solve the wiring issues that arise for controlling a large array of electrodes ${ }^{11-14}$. For example, optoelectronic tweezers (OET) has recently been shown to manipulate single cells and microscopic particles sandwiched between an ITO electrode and a photoconductive electrode created by direct optical images ${ }^{14}$. However, due to impedance mismatch, it is difficult to modulate the electric field strength in electrically insulating mediums, such as oils used in two phase droplet based microfluidic systems, on an OET device since all of the applied voltage drops across the oil layer even without light illumination.

Our group has previously reported a novel floating electrode optoelectronic tweezers (FEOET) mechanism ${ }^{15}$ enabling optical actuation of oil-immersed aqueous droplets using a circular laser beam with intensity as low as $4 \mu \mathrm{W} / \mathrm{mm}^{2}$. However, droplet movement is limited to a linear motion along the direction of electric field in the previous FEOET device, which strongly restricts its potentials in chemical and biological applications. Here, we demonstrate two-dimensional droplet manipulation functions on a
FEOET platform by utilizing diamond-shaped virtual electrodes patterned by a spatial light modulator. This opens up the possibility of massively parallel droplet manipulation on a featureless, silicon coated glass substrate using dynamic and reconfigurable optical images. Optically patternable virtual electrodes generate dielectrophoretic (DEP) forces to manipulate aqueous droplets containing chemical and biochemical contents and to perform various microfluidic functions such as two-dimensional transport, droplet merging and parallel processing of sixteen droplets.

\section{DEVICE STRUCTURE AND WORKING PRINCIPLE}

Figure 1 shows the schematic structure of a FEOET device. It is fabricated by coating a glass wafer with two featureless a-Si:H layers $\left(0.5-\mu \mathrm{m}\right.$ undoped and $\left.0.1-\mu \mathrm{m} \mathrm{n}^{+}\right)$. Two aluminum electrodes with a 6-cm lateral gap are deposited at the two edges of this device by e-beam evaporation and lift-off techniques. The Al electrodes also serve as an etching mask for removing the $\mathrm{n}^{+}$a-Si:H layer using Reactive Ion Etcher (RIE). The $\mathrm{n}^{+} \mathrm{a}-\mathrm{Si}: \mathrm{H}$ layer provides ohmic contacts with the Al electrodes. An open poly-dimethylsiloxana (PDMS) chamber housing aqueous droplets and immiscible oil (corn oil) is fixed on top of the undoped a-Si:H layer.

The previously reported FEOET device in our group was able to actuate an aqueous droplet by light-induced DEP forces with an intensity as low as $4 \mu \mathrm{W} / \mathrm{mm}^{2}$, an intensity promising a large area droplet manipulation platform with a regular and commercially available optical projector without extra optics components. However, DEP force induced on FEOET is dependent upon the direction of applied electric field and shape of optical virtual electrodes. In our previous experiment of droplet actuation using a circular light beam, DEP force induced in the direction parallel to
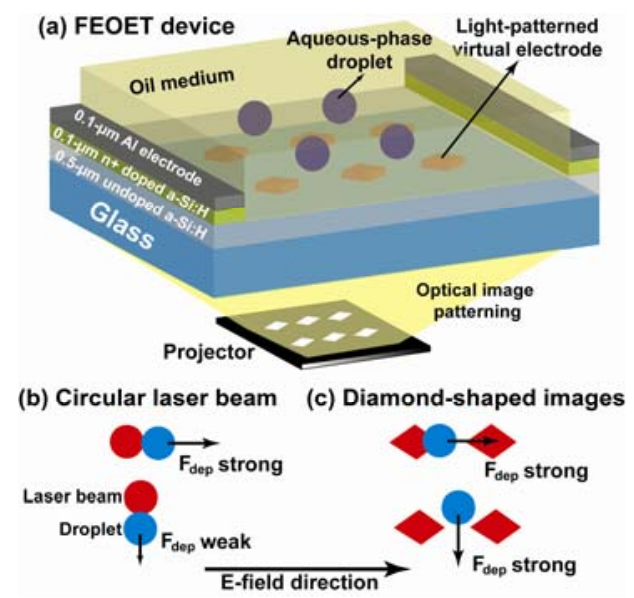

Figure 1: (a) Schematic structure of a FEOET device. (b) DEP forces induced by a circular light spot are weak in the direction perpendicular to applied field. (c) DEP forces induced by two diamond shaped virtual electrodes are strong in both parallel and perpendicular directions. 
the applied electric field is strong, but the force in the perpendicular direction is weak (Fig. 1b). This limits the droplet motion to one direction, which restricts FEOET's future applications.

To provide FEOET two-dimensional manipulation functions, we propose to trap a droplet between the gap of two diamond-shaped virtual electrodes as shown in Fig. 1c. This unique shape of virtual electrode is able to provide a droplet with the same order of magnitude DEP forces in both directions parallel and perpendicular to the applied field.

\section{SIMULATION RESULTS}

Numerical simulations of a three-dimensional electric field distribution in a FEOET chamber and on a droplet surface are performed using COMSOL Multiphysics 3.2. To reduce the simulation time, the device is simplified to two layers: a $5-\mu m$ thick undoped a-Si:H and a 350- $\mu \mathrm{m}$ thick oil medium. A $100 \mathrm{~V}$ DC bias is applied at the two end planes along the $\mathrm{x}$ direction, and a $300-\mu \mathrm{m}$ diameter aqueous droplet is loaded in a $1.4 \times 0.8 \mathrm{~mm}^{2}$ oil chamber. The dark conductivity of the homogeneous a-Si:H layer is assumed to be $10^{-8} \mathrm{~S} / \mathrm{m}$.

To compare the DEP forces induced by both a circular light beam and two diamond-shaped virtual electrodes, the circular light beam is assumed to have a $200-\mu \mathrm{m}$ full width half maximum (FWHM) spot size and it induces a peak photoconductive of $10^{-4}$ $\mathrm{S} / \mathrm{m}$ in the center. The same peak photoconductivity is also assumed in the case of diamond-shaped virtual electrodes with a $200-\mu \mathrm{m}$ separation gap. The Maxwell stress tensor has been applied for calculating DEP forces on droplets ${ }^{16}$.

\section{Case of a Circular Laser Beam Illumination}

Figure 2 shows the simulated electric field distribution around a droplet located at $150 \mu \mathrm{m}$ away from the center of a light beam along the direction parallel (Fig. 2a) and perpendicular (Fig. 2b) to the applied electric field.

Droplet actuation on FEOET results from the electrostatic dipole-dipole interactions between the droplet and virtual electrodes. Without light illumination, a droplet-induced dipole generates a symmetrical electric field distribution around a droplet surface, resulting in zero net DEP forces for transportation. To actuate a droplet, one can illuminate a light beam at one edge of a droplet to break this field symmetry around a droplet. As shown in Fig. 2(a), the electric field at the illumination side is greatly reduced, resulting in net DEP forces moving the droplet away from the light beam. The calculated DEP force is $F_{\text {dep }}=[-9.46,0.02,-2.87] \times 10^{-9}$ $N$, corresponding the forces in $\mathrm{x}, \mathrm{y}, \mathrm{z}$ directions, respectively. The $0.02 \mathrm{nN}$ force in the $\mathrm{y}$ direction comes from numerical errors.

Transporting droplet in the $\mathrm{y}$ direction, however, is not as effective as the on in the $\mathrm{x}$ direction using a circular light beam. Illuminating a droplet edge at the direction perpendicular ( $\mathrm{y}$ direction) to the electric field fails to transport the droplet since the electric field strength difference between the illuminated and un-illuminated sides on the droplet surface is too small as shown in Fig. 2(b). The calculated DEP forces in this situation is $F_{\text {dep }}=[-0.00$, $0.12,2.55] \times 10^{-9} N$. The y direction force is 78.8 times smaller than that of the $x$ direction force in Fig. 2(a). For this reason, a circular light beam is not able to provide the required shape of virtual electrodes for 2D transportation in FEOET devices.

\section{Case of a Paired Diamond-Shaped Virtual Electrode}

Figure 3 shows the simulation results of a droplet trapped by two diamond-shaped virtual electrodes. Without loading a droplet the electric field strength at the gap between these two electrodes is strongly enhanced (Fig. 3a at the top). When a droplet is loaded at $150 \mu \mathrm{m}$ away from the gap center along the $\mathrm{x}$ direction (Fig. 3b), the

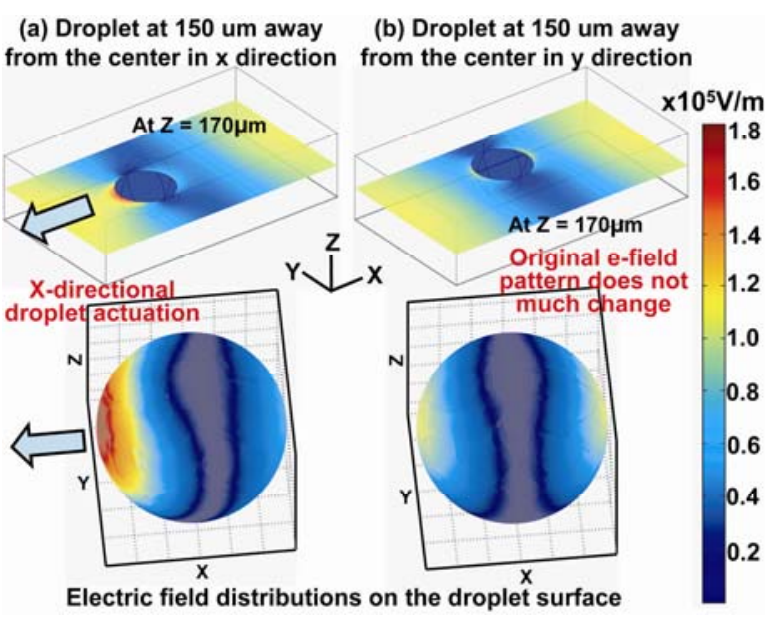

Figure 2: Numerical simulations of the electric field distributions by a circular laser beam illumination. (a) A droplet is located at 150 $\mu \mathrm{m}$ away from the center along the $\mathrm{x}$ direction. (b) A droplet is located $150 \mu \mathrm{m}$ away from the center along the $y$ direction.

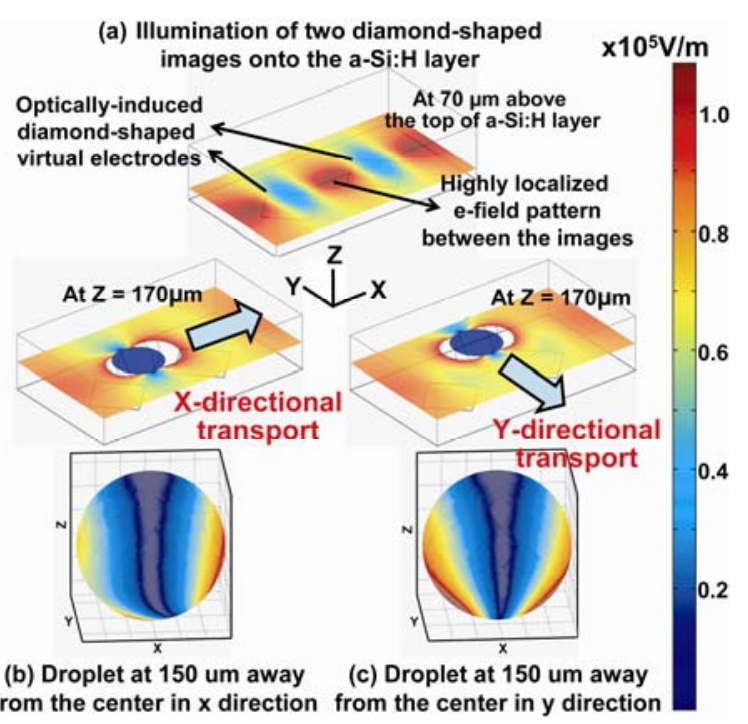

Figure 3: (a) Electric field distribution induced by two closely positioned diamond-shaped virtual electrodes separated by a $200 \mu \mathrm{m}$ gap. The strong electric field region is created at the gap. (b) A droplet is located $150 \mu \mathrm{m}$ away from the center along the $x$ direction. The unbalanced e-field pattern in the $x$ direction causes DEP force to drive and trap it to the right. (c) A droplet is located $150 \mu \mathrm{m}$ away from the center along the $y$ direction. The e-field pattern is symmetric in $x$ direction but asymmetric in the $y$ direction, producing a net DEP force to attract the droplet back to the center

enhanced electric field can generate DEP forces pulling the droplet back to the center of the gap. The calculated DEP forces are $F_{\text {dep }}=$ $[1.45,0.00,-2.48] \times 10^{-8} N$. The DEP force in the $\mathrm{x}$ direction is to the case of a circular laser beam.

For the situation of positioning a droplet $150 \mu \mathrm{m}$ away from the gap center along the y direction, the DEP force shows a dramatic increase compared to the $y$ direction force in the case of a circular light beam. As shown in Fig. 3(c), the calculated DEP forces in this situation are $F_{d e p}=[0.00,-0.64,-2.51] \times 10^{-8} N$. The y direction force 
is only 2.26 times smaller than the $\mathrm{x}$ direction force in the Fig. 3(b) situation. This value shows a dramatic improvement compared to the 78.8 times smaller in the circular light beam case. This y direction force is able to provide effective trapping and transporting of a droplet in the $y$ direction. This also realizes the two-dimensional droplet manipulation functions on FEOET devices.

\section{EXPERIMENTAL DEMONSTRATIONS}

With the capability of two-dimensional transport enabled by a paired diamond-shaped electrode, we have successfully demonstrated several important droplet manipulation functions on our FEOET platform, including continuous 2D transport, droplet merging, and parallel processing of 16 droplets.

Figure 4 shows the photo of a FEOET device with a $6 \times 6 \mathrm{~cm}^{2}$ active optical manipulation area. Two $\mathrm{Al}$ electrodes are applied by a $5 \mathrm{kV}$ bias to power the entire device. Although the voltage is high, but the power consumption of the whole device is $0.4 \mu \mathrm{W}$ in the dark state and less than $4 \mathrm{~mW}$ in the bright state due to the large electrical impedance of the oil and a-Si:H thin film $\left(\sim 10^{14} \Omega\right.$ in the dark state) in the lateral direction.

Figure 5 shows the video snapshots of a $1.47 \mathrm{~mm}$ aqueous droplet containing the Trypan blue dye being trapped at the gap between two diamond-shaped virtual electrodes and transported in both $\mathrm{x}$ and $\mathrm{y}$ directions by reconfiguring projected optical images. The droplet transport speed is $721 \mu \mathrm{m} / \mathrm{sec}$ in the $\mathrm{x}$ direction and 363 $\mu \mathrm{m} / \mathrm{sec}$ in the $\mathrm{y}$ direction. The two times difference between the speeds in the $\mathrm{x}$ and $\mathrm{y}$ direction matches well with the DEP force difference predicted in the simulation results in Fig. 3(b) and (c) using a $300 \mu \mathrm{m}$ droplet. Figure 6 shows that two 1-mm droplets containing yellow and green food coloring dyes are individually transported and merged together. When two aqueous droplets are driven close and aligned in the electric field direction, dipole-dipole interaction between two droplets induces electrocoalescence and combines two droplets together ${ }^{17}$.

Figure 7 demonstrates the flexibility and compatibility of FEOET devices with other microfluidic structures such as microwells. An example shown here is an array of PDMS microwells structure being positioned on top of a FEOET device. These wells are preloaded with aqueous solutions containing the Trypan blue dye and immersed in oil environment. A 1.28-mm de-ionized water droplet is transported by a paired diamond-shaped virtual electrode and delivered to the target well to dilute the dye concentration in that well.

The low light intensity requirement of FEOET and the two dimensional droplet transportation function realized by diamond-shaped virtual electrodes promise an FEOET platform

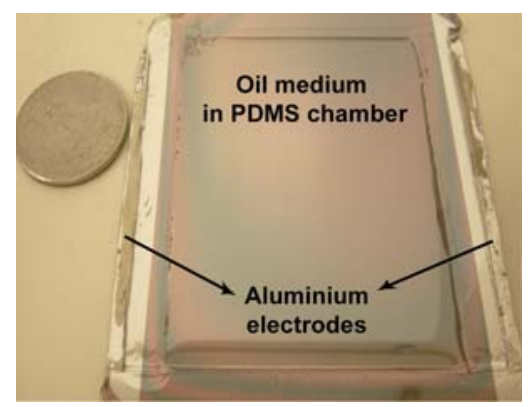

Figure 4: The photo of a FEOET device. The active area allowing optical manipulation of oil-immersed aqueous droplets is $6 \times 6$ $\mathrm{cm}^{2}$. A $5 \mathrm{kV}$ electrical bias is applied through the two Aluminum electrodes to power this device.

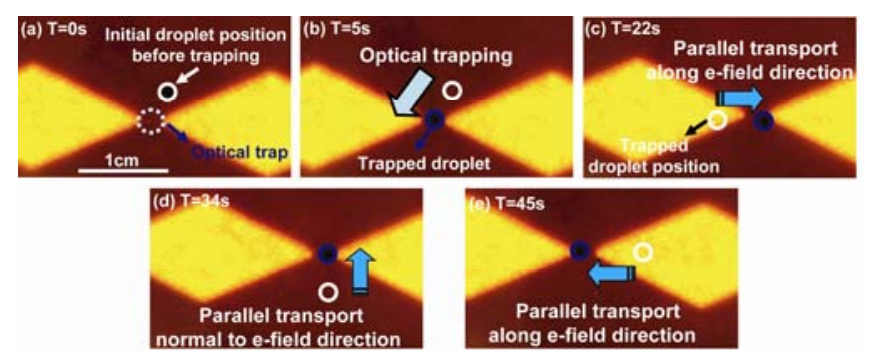

Figure 5: The video snapshots showing two-dimensional optical manipulation of a 1.47-mm droplet containing the Trypan blue dye on a FEOET device. The white and blue circles indicate droplet positions before and after movement (a) and (b) Two diamond-shaped virtual electrodes create strong electric field between the gap to trap the droplet. (c) The trapped droplet is transported to the right hand side along e-field direction. (d) The droplet is driven to the top side perpendicular to e-field direction. (e) It moves to the left hand side again.
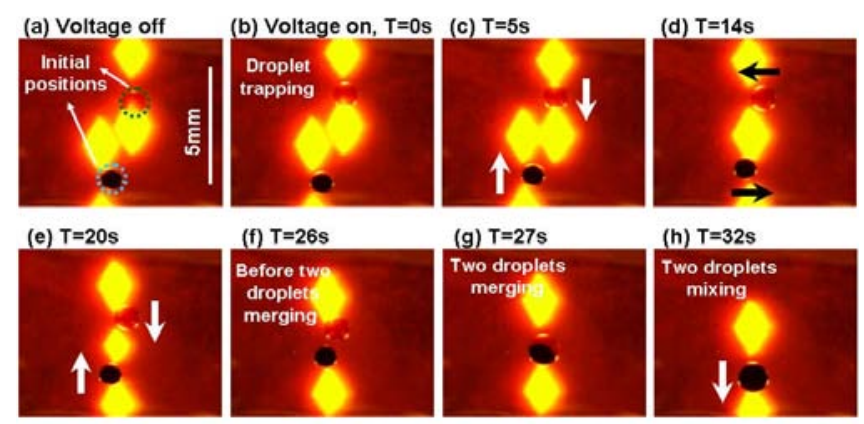

Figure 6: The video snapshots showing two 1-mm droplets containing different coloring dyes (yellow and green ones) are transported to merge.

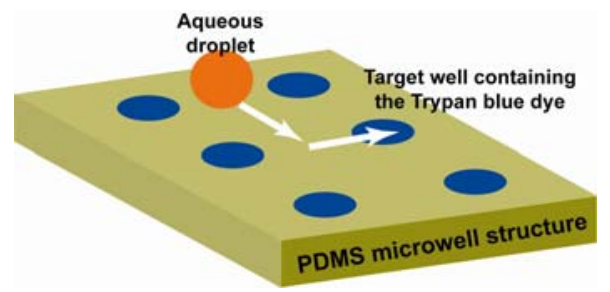

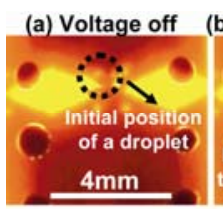
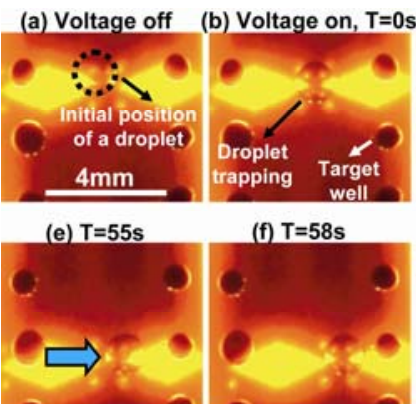

(f) $\mathrm{T}=58 \mathrm{~s}$

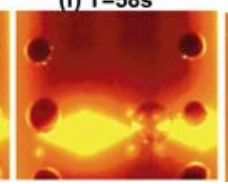

(c) $T=13 \mathrm{~s}$

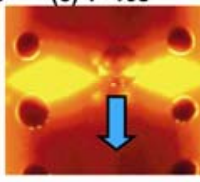

(g) $\mathrm{T}=60 \mathrm{~s}$
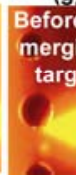

efore droplet

nerging into

target well (d) $\mathrm{T}=34 \mathrm{~s}$

Figure 7: Snapshots of the video showing the function of delivery of aqueous droplets to target wells among an array of PDMS wells positioned on FEOET. (a) and (b) A 1.28-mm de-ionized water droplet is optically trapped between two diamond-shaped images. (c) through (g) Two-dimensional transport of the droplet is achieved by the corresponding optical image motions. (h) Finally, the droplet is merged into the target well containing Trypan blue. 


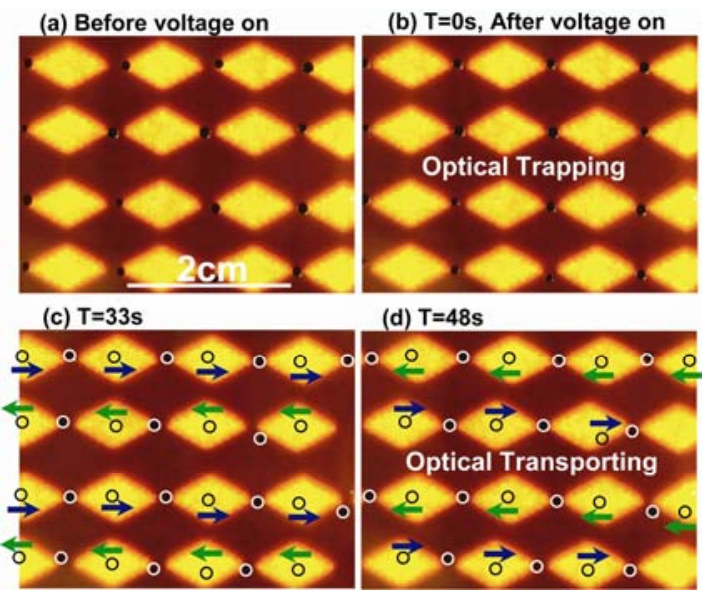

Figure 8: The video snapshots showing the large-scale, parallel manipulation of sixteen droplets of trypan blue dye with the range between 1.3-mm and 0.84- $\mathrm{mm}$ diameter. The black circles indicate the droplet position in previous step, while the white circles present the droplet position in the current step. a), (b) An array of diamond-shaped optical images generate sixteen traps and attract droplets between their gaps. (c),(d) Parallel transport of trapped droplets by dynamic optical images. (c) The droplets in the first and third rows are driven to the right side, while the droplets in the second and fourth rows are guided to the left.

capable of massively parallel processing a large number of droplets for high throughput chemical and biochemical screening applications. Figure 8 shows an example of parallel transporting 16 droplets. The droplet size in this experiment varies from 1.3-mm to 0.84-mm in diameter. Fig. 8(a) and (b) show the arrays of diamond-shaped virtual electrodes generate sixteen traps and position droplets between their gaps. In Fig. 8(c) and (d), the trapped droplets are transported to the left and right directions in parallel.

\section{CONCLUSIONS}

We report on an optically reconfigurable microfluidic platform enabling parallel processing of oil-immersed aqueous droplets on a photoconductive glass substrate using direct optical images. This platform is realized by using paired diamond-shaped optically patterned virtual electrodes that allow transporting droplets on a two-dimensional FEOET surface. With this platform, we have demonstrated several important droplet manipulation functions including continuous 2D droplet transport, droplet merging and parallel processing of 16 droplets. This FEOET system is also compatible with other microfluidic structures such as an array of microwells. Our platform promises a low cost, droplet-based, microfluidic system for large scale, multiplexed chemical and biochemical screening applications.

\section{ACKNOWLEDGEMENTS}

This project is supported by the NSF CAREER Award (ECCS-0747950), by the NIH Roadmap for Medical Research Nanomedicine Initiative PN2EY018228, and in part by NIH grants CA90571 and CA107300.

\section{REFERENCES}

[1] D. Albrecht, G. Underhill, A. Mendelson S. Bhatia, "Multiphase electropatterning of cells and biomaterials", Lab on a Chip, 7, 702-709 (2007).
[2] T. Tan, K. Hettiarachchi, M. Siu, Y. Pan, A. Lee, “Controlled microfluidic encapsulation of cells, proteins, and microbeads in lipid vesicles,” Journal of the American Chemical Society 128, 5656-5658 (2006).

[3] L. Li, J. Q. Boedicker, R. F. Ismagilov, "Using a multijunction microfluidic device to inject substrate into an array of preformed plugs without cross-contamination: Comparing theory and experiments”, Analytical Chemistry, 79, 2756-2761 (2007).

[4] D. Chen, L. Li, S. Reyes, D. Adamson, R. Ismagilov, "Using three-phase flow of immiscible liquids to prevent coalescence of droplets in microfluidic channels: Criteria to identify the third liquid and validation with protein crystallization", Langmuir, 23, 2255-2260 (2007)

[5] H. Song, R. F. Ismagilov, "Millisecond kinetics on a microfluidic chip using nanoliters of reagents," Journal of the American Chemical Society 125, 14613-14619 (2003).

[6] G. Cristobal, L. Arbouet, F. Sarrazin, D. Talaga, J. Bruneel, M. Joanicot, L. Servant, "On-line laser Raman spectroscopic probing of droplets engineered in microfluidic devices”, Lab Chip, 6, 1140-1146 (2006).

[7] M. Curcio, J. Roeraade, "Continuous segmented-flow polymerase chain reaction for high-throughput miniaturized DNA amplification”, Analytical Chemistry. 75, 1-7 (2003).

[8] J. Schwartz, J. Vykoukal, P. Gascoyne, "Droplet-based chemistry on a programmable micro-chip”, Lab on a Chip, 4, 11 (2004).

[9] Lee, C. C. et al., "Multistep synthesis of a radiolabeled imaging probe using integrated microfluidics”, Science, 310, 1793-1796 (2005).

[10] N. Manaresi, A, Romani, G. Medoro, L. Altomare, A. Leonardi, M. Tartagni, R. Guerrieri, "A CMOS chip for individual cell manipulation and detection,” IEEE Journal Of Solid-State Circuits 38, 2297-2305 (2003).

[11] A. Ohta, A. Jamshidi, J. Valley, H-Y Hsu, M. Wu, Appl. Phys. Lett. 91, 074103 (2007).

[12] A. Ohta, P. Chiou, H. Phan, S. Sherwood, J.Yang, A. Lau, H. Hsu, A. Jamshidi, M. Wu, J. Selec. Top. in Quantum electronics 13, 235-241 (2007).

[13] P. Y. Chiou, H. Moon, H. Toshiyoshi, C.J. Kim, M. C. Wu, "Light actuation of liquid by optoelectrowetting", Sensors and Actuators a-Physical 104, 222-228 (2003).

[14] P. Y. Chiou, A. T. Ohta, and M. C. Wu, "Massively parallel manipulation of single cells and Microparticles using optical images”, Nature, 436, 370-372 (2005).

[15] S-Y. Park, C. Pan, T-H. Wu, S. Kalim, M. Teitell, and P.Y. Chiou, "Floating Electrode Optoelectronic Tweezers (FEOET): A Novel Mechanism Enabling Optical Manipulation of Oil Immersed Aqueous Droplets", in Proceeding of the 11th International Conference on Miniaturized Systems for Chemistry and Life Sciences ( $\mu$ TAS) in Paris, France, Oct. (2007).

[16] C. Rosale, K. Lim, "Numerical comparison between Maxwell stress method and equivalent multipole approach forcalculation of the dielectrophoretic force in single-cell traps”, Electrophoresis (2005), 26, 2057-2065.

[17] J. Eow, M. Ghadiri, "Drop-drop coalescence in an electric field: the effects of applied electric field and electrode geometry", Colloids and Surfaces A: Physicochem. Eng. Aspects 219 (2003) 253-279. 